Article original

\title{
Quête spirituelle, résilience et Temps du Rêve chez les Aborigènes de Sydney
}

\author{
Spiritual quest, resilience and Dreaming in Sydney's Aboriginal population \\ E. Tasia ${ }^{1}$ \\ Laboratoire d'anthropologie des mondes contemporains (LAMC), ULB, institut de sociologie, 44, avenue Jeanne, CP 124, Bruxelles, Belgique
}

\section{N F O A R T I C L E}

Historique de l'article :

Disponible sur Internet le 28 octobre 2019

\section{Mots clés :}

Quête spirituelle

Temps du Rêve

Aborigène d'Australie

Culture urbaine

Résilience

Groupe thérapeutique

\begin{abstract}
R É S U M É
Contexte. - Ce texte s'inscrit dans une perspective d'anthropologie psychologique et s'intéresse à la continuité culturelle de pratiques de développement personnel et de soin parmi les populations aborigènes des grandes villes d'Australie.

Objectifs. - L'objectif de ce texte est double : (a) démontrer l'existence d'une réappropriation par certains Aborigènes contemporains urbains d'éléments culturels considérés, historiquement et anthropologiquement, comme " traditionnels ", notamment dans leurs liens avec la cosmologie du Dreaming ; (b) analyser l'impact de cette réappropriation culturelle sur la forme d'expression de la souffrance ressentie, à la fois individuelle et collective, et du soin qu'elle appelle nécessairement.

Méthode. - L'étude se base sur un corpus de données ethnographiques de première main, récolté lors des recherches doctorales de l'auteur : une observation participante de dix mois et demi au sein d'un groupe thérapeutique indigène situé dans la banlieue centrale de Sydney et une quinzaine d'entretiens semi-directifs avec les membres de ce groupe.

Résultats. - L'enquête démontre deux choses principales : (a) une réappropriation, par ces populations aborigènes urbaines, d'une conception altérée du corpus mythico-pratique qu'est le Dreaming ; (b) l'entreprise, par ces derniers, d'un processus thérapeutique dont l'objectif est d'engendrer un " allermieux ", sous l'axe d'un développement de soi. Celui-ci prend la forme d'une " quête spirituelle " et s'ancre pleinement dans cette version revisitée du Dreaming.

Conclusions. - Par l'exemple ethnographique qu'elle se propose d'exposer et analyser, cette étude cherche à mettre en avant une compréhension du thérapeutique comme étant indissociable, d'un point de vue pragmatique, du contexte culturel dans lequel celui-ci émerge. Au passage, elle informe sur l'ingéniosité culturelle et la résilience dont font preuve les Aborigènes des grandes villes de l'Australie pour parvenir à endiguer la souffrance générée par la colonisation.

@ 2019 Association In Analysis. Publié par Elsevier Masson SAS. Tous droits réservés.
\end{abstract}

\section{A B S T R A C T}

Context. - This article contains a discussion of cultural continuity between self-improvement and health practices amongst Aboriginal populations in large Australian cities, from a psychological anthropology perspective.

Goals. - There are two main purposes to this article: (a) showing how some contemporary urban Aborigines reappropriate cultural elements, historically and anthropologically considered as "traditional," especially concerning their links to the Dreaming cosmology; (b) analyzing how this cultural reappropriation impacts the way Aborigines express their pain, both individually and collectively. Method. - This study is based on first-hand ethnographic data collected during the author's doctoral research. The fieldwork consisted of a 10.5-month participant observation of an Aboriginal group

\footnotetext{
Adresse e-mail : etasia@ulb.ac.be

${ }^{1}$ Post-doctorant FNRS au Laboratoire d'anthropologie des mondes contemporains (LAMC), à l'université Libre de Bruxelles (ULB), Edgar Tasia est anthropologue et docteur en sciences Politiques et Sociales. Ses recherches portent principalement sur la santé mentale et le traumatisme. Il a réalisé ses recherches doctorales au sein d'un groupe thérapeutique aborigène de la banlieue centrale de Sydney

https://doi.org/10.1016/j.inan.2019.10.002

2542-3606/৫ 2019 Association In Analysis. Publié par Elsevier Masson SAS. Tous droits réservés.
} 
therapy circle in a suburb of Sydney and about 15 semi-structured interviews with some members of this group.

Findings. - This article presents two main findings: (a) the urban Aboriginal populations under consideration reappropriate an altered version of the myth and practices of the Dreaming and (b) their involvement in a therapeutic process aimed at allowing them to "feel better" takes the form of a spiritual quest framed in this altered conception of the Dreaming.

Conclusions. - Through the analysis of this ethnographic example, this research promotes an understanding of therapy as being intrinsically linked - from a pragmatic point of view - to the cultural background from which it emerges. Doing so, this article shows the cultural inventiveness and resilience demonstrated by Aborigines in Australia's large cities to contain the pain generated by colonization.

(ㄷ) 2019 Association In Analysis. Published by Elsevier Masson SAS. All rights reserved.

\section{Introduction : sémiologie contemporaine flottante d'une cosmologie millénaire}

La cosmologie des peuples aborigènes (généralement dénommée " Dreaming " ou " Dreamtime » en anglais ; « Temps du Rêve » en français) est principalement connue pour le paradoxe anthropologique qui la caractérise : d'une part, elle se veut théoriquement inchangée depuis plus de 60000 ans ; d'autre part, de nombreux chercheurs ont montré sa grande capacité plastique à s'adapter empiriquement aux pressions sociales et interrelationnelles des membres qui, par rituels interposés, la font vivre et exister (Glowczewski, 1989, 1991a, 2004 ; Dussart, 1988, 2000 ; Poirier, 2005). En cela, la cosmologie du Dreaming ${ }^{2}$ est donc particulièrement résiliente : changeante, mouvante mais fondamentalement " toujours la même "; celle-ci se réadapte afin de perdurer comme référence culturelle et spirituelle fondamentale pour les populations aborigènes contemporaines de l'Australie. Initialement, et au-delà de ses différentes variations locales (voir Dean, 1996) ou de ses problèmes de traduction et de compréhension (Glowczewski, 1991b ; Stanner, 1979 ; Wolfe, 1991), le Dreaming se réfère au temps primordial des héros totémiques ayant, par leurs passages sur la terre, formé le paysage environnemental ainsi que les différents chemins et lieux sacrés - les dreaming tracks - qui le constituent. En tant que métaphysique, le Dreaming fonctionne donc comme un " passé mythologique [qui] est vivant et pertinent dans le présent " (Berndt \& Berndt, 1965, p. 188), reliant les Aborigènes à ces héros totémiques et aux restes des éléments du monde (plantes, animaux, autres humains, lieux, etc.), puisque ceux-ci sont considérés comme des dérivés de la substance initiale de ces déités. Cette cosmologie fonctionne donc comme un corpus mythico-pratique organisant, à la fois la vie spirituelle et mystique, et à la fois la vie sociale et politique des populations aborigènes du pays.

Dans l'environnement urbain actuel, la reconquête du Dreaming par les populations aborigènes des grandes villes de l'Australie est due à deux facteurs sociologiques différents et pourtant liés entre eux par un même contexte postcolonial. D'abord, (a) une mutation dans les fondements épistémologiques qui permettent de définir l'identité aborigène d'un point de vue subjectif (Keen, 1988). D'une part, comme l'a notamment montré Lambert-Pennington (2012),

\footnotetext{
${ }^{2}$ Par souci de cohérence, dans cet article, c'est la dénomination anglo-saxonne de "Dreaming " qui sera privilégiée. Deux raisons à cela : d'abord, car c'est celle qui est la plus couramment usitée par les individus que nous avons rencontrés. Ensuite, parce que c'est la dénomination choisie par W. Stanner (1979) qui, à juste titre, considère le Dreaming comme "bien plus compliqué, d'un point de vue philosophique, que l'on a pu l'imaginer initialement " (Stanner (1979), p. 24). Or, c'est bien de cette complexité que découle la plasticité de ce corpus mythicopratique - plasticité que l'on voudrait illustrer à l'aune d'un exemple urbain contemporain
}

c'est aujourd'hui dans le sentiment, la sensation (feeling) d'appartenir à une telle communauté que se cristallise l'appartenance affective ${ }^{3}$. D'autre part, c'est en s'appropriant à l'échelle individuelle et communautaire " la " culture aborigène ${ }^{4}$ - culture fondamentalement « mythologisée " (Cowlishaw, 2010, 2011) par le discours public et les politiques multiculturelles mises en place par le gouvernement australien (Povinelli, 2002 ; voir aussi Henry, 2014) - que les individus concernés s'accaparent cette identité. Or, une telle culture aborigène unique se veut nécessairement lissée et recentrée autour d'éléments emblématiques, symboliquement forts (voir Tasia, 2018b, pp. 141-146). Parmi ces derniers, le Dreaming, dans sa dimension " spirituelle ", occupe une place centrale (Grieves, 2006 ; 2009 ; Pattel-Gray, 1996). C'est que (b) l'imaginaire et la symbolique, écologiste notamment, que charrie le Dreaming, est particulièrement propice à faire écho à certains jeux de langage "New Age " de la société occidentale contemporaine (Neuenfeldt, 1998 ; Sutton, 2010). Le « message » à la fois spirituel, écologique, altruiste, communautaire et " authentique " que celuici semble posséder permet en effet au Dreaming de fonctionner comme une sorte de réservoir sémantique et symbolique dans lequel les différents acteurs du corps social viennent puiser les raisons de leurs actions, de leurs préceptes moraux ou de leurs choix politiques. Autrement dit, par l'aplatissement anthropologique dont elle fait l'objet, la cosmologie du Dreaming s'autorise une mutation sémiologique permettant à certains de ses préceptes symboliques et culturels de se fondre dans plusieurs jeux de langage de la société contemporaine ; les plus importants d'entre eux étant sans doute les jeux de langage du "développement personnel " et du " soin " (Tasia, 2018b).

Ainsi, pour plusieurs Aborigènes de la banlieue centrale de Sydney, la souffrance quotidienne endurée, souffrance qui incombe au traumatisme intergénérationnel découlant du processus colonial (voir Atkinson, 2002), est l'occasion d'entamer

\footnotetext{
${ }^{3}$ Nous ne traitons pas ici de l'appartenance effective à la communauté aborigène du pays qui, quant à elle, est réglementée et dépend de trois facteurs : (a) le lien biologique avec des ancêtres aborigènes, (b) l'appartenance - validée - à une communauté aborigène et (c) l'affirmation autodéterminée d'identification. Pour une analyse détaillée de cette question, voir notamment Kowal (2017). Plutôt, nous cherchons à saisir ce par quoi l'affirmation d'une telle identité - aborigène - passe lorsqu'elle s'affirme intimement et publiquement.

${ }^{4}$ Traditionnellement, le terme de "Loi " est généralement préféré au terme de " culture " par les populations aborigènes de l'Australie (voir Glowczewski, 1991a). $\mathrm{Si}$ le terme de " culture " ne s'est imposé dans les milieux aborigènes qu'à partir des années 80 , cela ne s'est fait qu'à la suite d'une politique multiculturelle et patrimoniale volontairement adoptée et astreinte par le gouvernement australien (Glowczewski, com. pers.). Dans la présente étude, ce sont donc les acteurs euxmêmes qui mentionnent le terme de " culture " ou de " racines culturelles " pour désigner ce corpus socioculturel de croyances et de traditions auquel ils se sentent liés (" notre culture »), et duquel ils se sentent cependant éloignés, voire séparés, tout en cherchant à se le réapproprier.
} 
un parcours initiatique particulier : celui d'un " voyage thérapeutique " (healing journey) passant par le développement de soi-même et l'accroissement de ses capacités de résilience ${ }^{5}$ tout en se reconnectant à ses " racines culturelles " - dont le Dreaming fait nécessairement partie. C'est à un tel parcours que voudrait s'intéresser cet article. L'objectif de ce texte sera donc double. D'une part, il s'agira de démontrer, en s'appuyant sur plusieurs données ethnographiques de première main, l'existence d'une réappropriation par certains Aborigènes urbains contemporains de plusieurs éléments culturels considérés, historiquement et anthropologiquement, comme " traditionnels "; d'émettre l'hypothèse d'une concordance de pratiques et de discours, autorisant la continuité, sous de nouvelles modalités, d'une forme de " quête spirituelle " $(\mathrm{QS})^{6}$ culturellement ancrée dans l'esprit du Dreaming. D'autre part, analyser l'impact de cette réappropriation culturelle sur la forme d'expression de la souffrance ressentie et du soin qu'elle appelle nécessairement. Pour ce faire, nous procéderons en deux temps : nous reviendrons d'abord sur le contexte sociopolitique global autorisant l'émergence de la QS en tant que démarche de sens pour certains Aborigènes de Sydney. Tout en présentant notre cas d'étude empirique (le Gamarada), nous analyserons ainsi brièvement plusieurs jeux de langage traversant la société australienne contemporaine (le " développement personnel " et le " traumatisme intergénérationnel ", notamment) qui participent à un tel résultat. Nous décortiquerons ensuite de manière analytique les divers éléments structuraux qui composent la QS (le contexte culturel, les dispositions des acteurs et les techniques utilisées) en les confrontant aux données empiriques.

\section{Du développement personnel à la quête spirituelle}

De par le contexte postcolonial que nous évoquions plus haut, nombreux sont les Aborigènes contemporains qui souffrent de problèmes de santé mentale (Atkinson, 2002 ; Australian Institute of Health and Welfare [AIHW], 2015 ; Burbank, 2011). Pour y remédier, et tenter d'enrayer le cercle vicieux de la reproduction de comportements (auto-)destructeurs généralement considérés comme découlant d'un " traumatisme intergénérationnel " qui remonte à la colonisation (Atkinson, 2002), certaines initiatives locales se sont peu à peu constituées. C'est le cas du Gamarada, ce groupe de parole aborigène qui se réunit chaque semaine depuis plus de dix ans dans une salle du quartier de Redfern ${ }^{7}$. Gratuit et non-obligatoire, ce groupe de parole se présente à ses membres comme un programme à vocation thérapeutique destiné principalement aux Aborigènes (bien que formellement ouvert à tous) souhaitant opérer des changements dans leur vie. Gamarada

\footnotetext{
${ }^{5}$ Le terme de " résilience " sera entendu dans ce texte comme " capacité à réussir, à vivre et à se développer positivement, de manière socialement acceptable en dépit du stress ou d'une adversité qui comporte normalement le risque grave d'une issue négative " (Cyrulnik, 2002, p. 8). La résilience sera donc perçue comme cette capacité à " rebondir " positivement à la suite d'un " traumatisme ", c'est-à-dire à la suite d'un choc psychologique découlant d'une expérience personnelle particulièrement désagréable, stressante et perturbante (Freud, 2010 ; Ferenczi, 2010), ou d'un état de stress quotidien structurellement généré par le contexte sociohistorique (Burbank, 2011).

6 Dans la suite du texte, il sera fait usage d'abréviations pour dénommer les quelques concepts-phares dont il est question : " quête spirituelle ", " développement personnel " et " traumatisme intergénérationnel "; respectivement, "QS ", " DP » et « TI ». Cette démarche a d'abord pour objectif d'alléger la lecture. De plus, cette astuce syntaxique permet ensuite de marquer une différence sémantique entre, d'une part les catégories émiques dont usent les acteurs étudiés, et d'autre part les concepts étiques que l'on cherche ici à systématiser.

Quartier du centre de ville de Sydney et haut symbole de l'activisme politique aborigène (voir McComsey, 2013). Les données empiriques mobilisées dans cette étude ont été récoltées au cours de mes recherches doctorales. Ces données sont issues d'une observation participante de dix mois et demi au sein de ce groupe de parole aborigène qu'est le Gamarada. Pour plus de détails concernant mon entrée sur ce terrain spécifique, voir Tasia (2016).
}

signifie " amis partageant un but commun " (" friends with a purpose ") en langue $\mathrm{Gadigal}^{8}$. En son sein, on pratique divers exercices ayant pour objectif d'engendrer, chez les pratiquants, de la résilience (voir Tasia, 2018a, b). La constitution du groupe est extrêmement plastique : le nombre de participants oscille entre cinq et 12 , et se situe généralement autour de huit membres; la majorité de ceux-ci sont des hommes aborigènes, âgés entre 30 et 60 ans. Chaque séance possède donc une composition unique, menant à une dynamique de groupe toujours singulière. Cependant, la structure du programme - bien que flexible - reste généralement constante d'une séance à l'autre. Ces dernières se déroulent en plusieurs étapes (appelées " tâches » ou " exercices ») et culminent dans la pratique d'un exercice de méditation guidée traditionnelle, le Dadirri. La disposition des lieux s'apparente à un cercle de chaises organisées autour d'un drapeau aborigène posé sur le sol. Réunis pendant deux heures, les participants sont amenés à partager les uns avec les autres plusieurs fragments de leurs histoires personnelles et à former ainsi un collectif de soutien affectif (Tasia, 2018a). Ce faisant, ils sont invités à entamer un " travail de soi " au sein duquel chacun peut engendrer de la résilience.

Pour les membres les plus convaincus par le programme, l'objectif de ces réunions se situe cependant quelque peu ailleurs : c'est dans l'opportunité qu'offre le dispositif qu'est le Gamarada d'entamer un " voyage " (journey), un "voyage thérapeutique " (healing journey), une "quête spirituelle " (spiritual journey) renouant avec certains éléments culturels constituant de ce type de pratiques que se trouve l'intérêt d'un tel programme. En effet, pour de nombreux membres, le Gamarada est avant tout un lieu où un " esprit " particulier (the Gamarada spirit) peut être trouvé et absorbé. Cet " esprit ", constitué par la " magie " du collectif renouvelé chaque semaine (voir Tasia, 2018b), fonctionne, pour les membres, comme une sorte de réservoir énergétique dans lequel ces derniers viennent puiser ce dont ils ont besoin pour "se développer ", " accroître " leur connaissance d'eux même. Ainsi, pour bon nombre de ses membres, le Gamarada joue le rôle de plateforme, de dispositif thérapeutique où le " soin " (healing) passe nécessairement par un cheminement spirituel ; où la souffrance subjectivement ressentie et collectivement vécue est l'occasion d'entamer une quête vers une meilleure version de soi-même.

À bien des égards, l'enchevêtrement des rhétoriques du " soin ", de l'épanouissement de l'individu et de la QS n'a rien de surprenant. Il trouve ses origines contemporaines dans le jeu de langage du " développement personnel " (DP), jeu de langage particulièrement pertinent pour les sociétés individualistes où l'autonomie est devenue une " condition pour l'action " (Ehrenberg, 2012, 2018; Marquis, 2014). Et si un tel jeu de langage prend racine dans un corpus de traditions bien éloignées des cultures aborigènes (voir Marquis, 2016 ; Requilé, 2008), celui-ci possède pourtant plusieurs caractéristiques constitutives de son discours qui lui permettent de franchir aisément la frontière culturelle ; car, d'une part, le contexte sociopolitique de l'Australie contemporaine - empreinte d'une idéologie individualiste et néolibérale puissante (Povinelli, 2010 ; Stratton, 2011) - le lui autorise et, d'autre part, ces mêmes caractéristiques relèvent d'une certaine universalité (Torrance, 1994). Inscrit dans un " psychocentrisme " qui, historiquement et sociologiquement, le dépasse (Rimke, 2012), le jeu de langage du DP se caractérise par un nouveau type de rapport au psychisme et à l'intériorité, ainsi qu'à la psychothérapie : ce rapport prend la forme d'une quête intérieure qui, passant par des pratiques introspectives spécifiques (médiations, exercices de respiration, imagination active, etc.), se doit de mener à l'épanouissement, à l'accroissement des capacités

${ }^{8}$ Les Gadigals sont un groupe aborigène du peuple Eora, qui, originairement, résidaient sur le territoire où s'est constitué le centre-ville de Sydney. 
psychiques de l'individu. Ainsi, le jeu de langage du DP, s'appuyant sur toute une série de pratiques hétéroclites, forme la trame d'une tendance sociale générale : celle de concevoir le malheur comme un état momentané, potentiellement surmontable ; que la mise en application rigoureuse d'une série d'exercices visant à " accroître " l'individu, permet d'enrayer.

Épistémologiquement, le jeu de langage du DP entretient donc de nombreuses affinités avec l'idée de QS (Garnoussi, 2013 ; Zsolnai \& Flanagan, 2019). En effet, pour Robert Torrance, une telle quête peut être comprise comme " un effort délibéré de transcender, au moyen d'une transformation de soi, les limites d'un donné, dans le but d'atteindre une portion d'un potentiel sans borne au travers de la poursuite d'un objectif fondamentalement inaccessible » (1994 p. xii). Poursuivre une QS, c'est donc chercher à dépasser un certain état de soi-même, à transcender celui-ci, dans le but de viser - plus qu'atteindre - un objectif supérieur ; c'est, en quelque sorte, parcourir sur le long cours, l'asymptote de sa propre potentialité. Entamer une telle quête, ce n'est donc pas " simplement » chercher à aller mieux; ce n'est pas non plus tenter de dépasser ponctuellement un état particulier (en cela, la QS telle qu'elle est entendue dans ce texte ne peut pas être résumée à une démarche exclusivement thérapeutique) - de telles propositions étant plutôt des moyens d'y entrer que des moyens de la vivre. Réaliser une telle quête, c'est plutôt renouer, par des voies culturellement modulées, avec une tendance fondamentalement humaine : celle d'outrepasser sans cesse les limites d'un état particulier en vue d'en élargir les frontières connues ; celle d'une créativité nécessaire toujours renouvelée (Torrance, 1994, p. 57).

Grâce aux travaux de plusieurs anthropologues du siècle passé, de telles entreprises de " transcendance de soi-même " au sein des cultures aborigènes traditionnelles, nous sont relativement bien connues (Berndt, 1947 ; Elkin, 1977, 1986 ; Spencer \& Gillen, 1898). Pleinement ancrées dans la cosmologie du Dreaming, ces entreprises répondent aux mêmes schèmes structuraux de " développement psychique " que la QS telle que nous l'avons délimitée. Adolphus Elkin, traitant de la question des hommes aborigènes de " haut rang " (Aboriginal men of High Degree) relève ainsi, sur tout le territoire australien, plusieurs phénomènes correspondant au dépassement des limites psychiques de l'individu : les initiés concernés, après être passés par une série d'épreuves " faisant " d'eux ("making") des " hommes d'esprit " ("clever men"), obtiennent généralement des pouvoirs psychiques leur permettant de contrôler le temps, l'espace, la matière et la mort (1977, p. 32). Pour atteindre un tel développement psychique, ces derniers se doivent de " mourir " à leur état initial pour ensuite " renaître ", transformés de "l'intérieur » (Elkin, 1977, p.20) : au cours d'une telle renaissance, les individus se voient alors dotés d'une nouvelle intériorité constituée - selon les différentes régions - de cristaux de quartz, de pierres magiques (australites), des coquillages sacrés ou de substances magiques (Elkin, 1977, pp. 20-29). Dans tous les cas, c'est grâce à l'aide et au contact des héros totémiques du Dreaming que de telles transformations sont rendues possibles.

Pour certains membres du Gamarada, dont tous cherchent à renouer avec « leur » culture, évoquer l'idée d'une $Q S$ n'est donc pas anodin tant une telle démarche s'inscrit à la fois dans l'air du temps (par les accointances qu'elle entretient avec le jeu de langage du DP) et à la fois dans un corpus de traditions aborigènes des plus authentiques (par les liens qu'elle entretient avec le Dreaming). Mais, pour qu'une telle démarche de $\mathrm{QS}$ puisse néanmoins faire sens dans le contexte actuel, trois éléments restent, d'un point de vue socio-anthropologique, nécessaires : (I) un contexte, (II) des dispositions et (III) une (ou des) technique(s) (Fig. 1). Que le jeu de langage du DP soit le terreau favorable d'une renaissance de la dimension spiritualisante des cosmologies aborigènes sous la forme d'une QS n'est pas suffisant pour en comprendre la réappropriation profonde par certaines populations aborigènes urbaines du pays. C'est donc à l'étude de ces trois éléments que l'on voudrait maintenant s'attarder car c'est à l'intersection de ces derniers que se laissent découvrir le sens et l'usage de l'expression "quête spirituelle ", ainsi que l'inscription d'une telle démarche dans la sémiologie urbaine contemporaine du Dreaming.

\section{Le traumatisme intergénérationnel et le Dreaming comme contexte}

Nous l'avons dit, pour les membres du Gamarada, le malheur et la souffrance endurés au quotidien incombent à une raison précise : le traumatisme intergénérationnel (TI). Ce jeu de langage du " traumatisme " (voir Tasia, 2018b) - appelant directement à lui celui du " soin " - n'est évidemment pas propre au Gamarada mais, plutôt, traverse l'entièreté de la société australienne lorsqu'il s'agit de caractériser la situation désastreuse des Aborigènes - en tant que peuple - tant du point de vue socio-économique que du point de vue psychologique ou hygiénique (AIHW, 2015 ; Atkinson, 2002 ; Atkinson, Nelson, \& Atkinson, 2010 ; Burbank, 2011 ; Murphy, 2011 ; O'Loughlin, 2009 ; Lavarch, 1997). En son sein, se cristallise une " guerre de l'histoire " (Attwood, 2005 ; Macintyre \& Clark, 2005) des fondations de l'Australie contemporaine cherchant à rétablir la vérité de l'épisode colonial (Moses, 2012 ; Reece, 1974 ; Reynolds, 1979), mais également, sur un plan plus pratique, celle de ses conséquences postcoloniales, et donc des souffrances et des abus subis par les populations aborigènes pendant plus de deux siècles (Atkinson, 2002 ; Attwood, 2005). Pour les membres du Gamarada donc, le TI se trouve à la source des divers problèmes et malheurs rencontrés en tant qu'Aborigène dans la société contemporaine. Parmi les diverses trajectoires de vie de ses membres, nombreuses sont les brèches (comme, par exemple, l'alcoolisme, l'usage de drogues, les abus physiques et psychologiques, les déceptions amoureuses, etc.) qui peuvent être identifiées (voir Tasia, 2018b). Cependant, de telles brèches brisures ponctuelles et idiosyncrasiques dans la trajectoire de chacune et chacun - ne peuvent apparaître que comme le reflet, l'incarnation pragmatique, d'une brèche plus profonde, toujoursdéjà-là, celle du TI. La " nature " d'une telle brèche va alors venir conditionner l'attitude des membres vis-à-vis du " soin " recherché. Fondamentalement insolvables, irréconciliables, les causes profondes du malheur de chacun obligent à entreprendre une démarche thérapeutique particulière : celle d'un cheminement, d'un parcours vers un " aller-mieux " hypothétique, intrinsèquement inaccessible ; celle d'une poursuite vers un passé culturel, si pas détruit, du moins démembré, décousu et déstructuré par plus de deux siècles de violence coloniale ; celle d'une double quête donc, à la fois orientée vers le passé collectif (" la " culture aborigène) et à la fois tournée vers un futur individuel - une meilleure version de soi-même, délestée du poids du TI et " renforcée " (empowered) par son parcours - tous deux intrinsèquement inaccessibles mais néanmoins désirables. De ce fait, cette quête engendre des effets pragmatiques de résilience. Dans ce contexte socio-anthropologique, le Dreaming dans sa version contemporaine revisitée (voir plus haut), devient luimême objet contextuel puisque c'est notamment au travers lui, de la recherche d'une " connexion " avec sa substance, que vont s'entreprendre les démarches thérapeutiques. En effet, au sein et en dehors du cercle qui compose le Gamarada, c'est notamment par une réappropriation de la mytho-pragmatique constitutive du Dreaming que va s'opérationnaliser la reconnexion des membres avec « leur " culture, avec les « racines culturelles " tant recherchées et désirées ; c'est notamment par la pratique de certaines techniques de rêverie, par l'échange d'histoires mythiques et par la recherche de signes liés au Dreaming que va s'effectuer cette double quête d'un passé collectif et d'un futur individuel (voir ci-après, Section 3 ). 


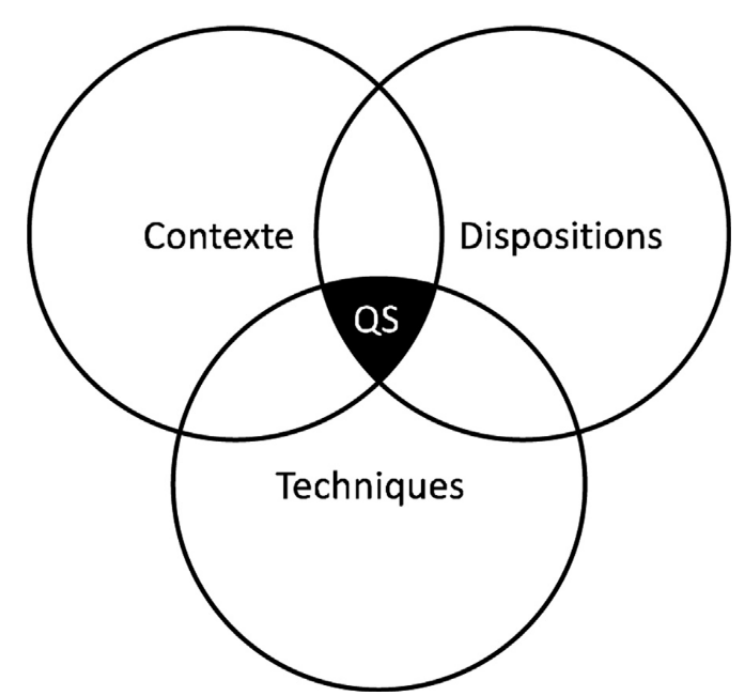

Fig. 1. La quête spirituelle trouve le sens de sa démarche à l'intersection de trois éléments socio-antgropologiques : le contexte dans lequel elle s'inscrit, les dispositions de ses acteurs et les techniques utilisées par ces derniers pour l'opérationaliser.

\section{L'apérité et l'ouverture perceptuelle comme dispositions principales}

Si le contexte susmentionné nous permet de comprendre le sens d'une démarche (celle de la QS), sa rapide étude laisse pourtant dans l'ombre un élément trivial de celle-ci : tous ne s'y attellent pas. Pour le faire, il faut nécessairement être réceptif à sa trajectoire asymptotique, son architecture cognitive et son vocabulaire. Autrement dit, si un environnement particulier est bel et bien nécessaire pour permettre à la QS d'émerger (ici, les jeux de langage du DP et celui du TI), il n'est absolument pas suffisant ; certaines dispositions sont requises. Parmi ces dernières, une en particulier nous semble cruciale : celle de parvenir à voir dans la contingence du quotidien les signes d'un destin en train de se réaliser ; celle d'une perception du monde comme monde qui parle ; celle d'être capable de tisser en trame sensée des éléments contingents, hétérogènes et hétéroclites. Pour Katie Glaskin (2015), le contexte culturel proprement aborigène qu'est le Dreaming, autorise les Aborigènes à faire preuve d'une telle capacité cognitivo-affective "d'ouverture perceptuelle " (percepperceptual openness). Pour cette auteure, celle-ci prend la forme d'une habileté prégnante à établir des associations et des connexions entre de nouveaux éléments et d'autres déjà connus, à " reconstruire la mémoire d'évènements passés " (Strauss \& Quinn, cité dans Glaskin, 2015, p. 665) à partir de cette liaison, à combler de sens les trous laissés par une information ambiguë tout en octroyant à l'ensemble du processus un surplus d'éclaircissement face à la situation présente. Ainsi, par l'inter-connectivité qui le caractérise tout particulièrement en tant que cosmologie mais également en tant que système d'organisation sociale (Poirier, 2005), le Dreaming favoriserait chez ses adeptes une habileté à établir des connexions et des associations créatives. Cette capacité " d'ouverture perceptuelle " se rapproche à bien des égards de ce que Nicolas Marquis a qualifié, dans son étude des lecteurs de livres de développement personnel, "d'apérité " (2014). Pour le sociologue belge, l'apérité (qui vient du latin aper, ouvert), c'est " cet état, cette disposition qui permet au lecteur de créer des éléments pertinents voire personnalisés dans les messages du DP " (Marquis, 2014, p. 130). C'est, au fond, cette disposition d'ouverture qui permet " d'être alerte par rapport à tout ce qui pourrait faire sens " (Marquis, 2014). Or, que celle-ci soit appelée " ouverture perceptuelle " ou " apérité ", c'est effectivement sur ce genre de dispositions particulières que les membres du Gamarada s'appuient lorsqu'ils se réunissent au sein du cercle. En effet, ces derniers sont amenés, une fois assis les uns en face des autres, à se nourrir des narrations des autres pour y trouver un sens (" thérapeutique ") tout personnel ; ils sont encore amenés à percevoir dans les détails du monde des éléments leur indiquant la voie (de " développement ") à suivre ; ils sont enfin amenés à connecter plusieurs fragments culturels issus des cultures aborigènes traditionnelles (comme, par exemple, le Dadirri) avec " leur " culture, avec la spiritualité aborigène lissée ou la mythopratique du Dreaming réinterprétée (Tasia, 2018a, 2018b). Voici, à titre d'exemple, les déclarations d'un membre actif du groupe à propos de sa fréquentation du Gamarada :

Tu sais, grandir pour moi a été... ça été difficile : chômage (pension welfare), devoir me soucier tout le temps du prochain repas, devoir toquer à de nombreuses portes car les factures n'arrivaient pas à se payer... J'ai dû arrêter l'école... Je suis passé quelques fois devant le juge alors que j'étais encore qu'un gosse... Et, tu sais, ce genre de trucs, c'était. . . ça été mon initiation. Grâce à cette expérience, j'arrive à me connecter (relate to) à pas mal de problèmes vécus par les autres. Donc le Gamarada, pour moi, ça m'aide à rester connecté à l'esprit (keep me connected to the spirit)... C'est une façon de rester connecté à ma culture. Ça donne un sens à ma vie. Ça me donne l'occasion d'être une partie de quelque chose de plus grand. C'est beaucoup de choses, mais principalement, c'est une connexion culturelle, un sens de la responsabilité et une source de développement personnel. Sans ce groupe, je ne serais sans doute pas moi-même aujourd'hui.

Seulement alors les membres sont-ils capables de voir dans l'entreprise de la transcendance du soi-même, à la fois un élément appartenant à la structure d'une thérapie spécifique (considérée, alors, comme adéquate et efficace), et à la fois comme une démarche personnelle nécessaire permettant d'accéder à une connaissance supérieure.

\section{Le partage d'histoires mythologiques, le Dadirri et la quête de signes comme techniques}

Si la rencontre entre un environnement adéquat et des dispositions adéquates permet de faire émerger un sens intrinsèque à la QS pour l'individu en recherche d'un tel sens, seule une (ou des) technique(s) adéquate(s) permet(tent) de l'opérationnaliser et de l'en distinguer d'un " simple " travail de soi. En effet, dans la dimension technique d'une pratique de QS se précipitent et se condensent divers éléments sociaux octroyant à cette dernière le droit de s'inscrire à la fois dans le registre du profane (le développement de soi) et du sacré (la transformation de soi), par le truchement de la référence culturelle adéquate (la transcendance de soi par la culture). Là, dans cette techno-logie, se glisse alors le sens historique des interactions sociales passées d'un collectif, se cristallise la dimension sociohistorique d'un milieu et d'une culture spécifique, faisant d'une séquence de gestes neutres, un rituel sensé au pouvoir performatif inexorable. Pour les membres du Gamarada, c'est une combinaison de trois pratiques différentes qui joue ce rôle de technologie culturelle adéquate (c'est-à-dire sensée, efficace et performative pour l'opérationnalisation de la $\mathrm{QS}$ ) : (a) le partage des récits mythologiques, (b) la pratique du Dadirri et (c) la recherche consciente et quotidienne de signes.

Au cours d'une séance typique du Gamarada, les membres sont amenés à parler d'eux-mêmes, à se mettre en mots afin d'entamer un travail de soi ; ils sont également invités à écouter attentivement les récits des autres afin d'y puiser des éléments pertinents pour leur propre développement (Tasia, 2018a, 2018b). Pour scander ces échanges - souvent lourds en drames humains - 
et ainsi dynamiser le protocole, les membres s'autorisent communément (a) des "digressions " prenant, parfois, la forme de partages de récits mythologiques. Au cours de ces dernières sont alors narrées les histoires fondatrices de plusieurs héros totémiques associés au Dreaming (tel que Baiame ou le Rainbow Serpent, par exemple). Ces narrations, effectuées dans le cadre spécifique que représente le Gamarada, sont l'occasion d'une "re-connexion " avec " leur " culture et plus particulièrement avec la dimension mythique du Dreaming. Que l'origine culturelle des différents héros totémiques appartienne à des cultures aborigènes parfois fort éloignées n'a que très peu d'importance : seul compte le rôle de " mythe fondateur " (Freud, 2015) que jouent ses histoires pour le groupe et ses membres ; seul compte le lien retrouvé, régénéré par l'écoute attentive de ces histoires, entre ces individus et le Dreaming. À cette pratique, vient s'en greffer une autre : celle du (b) Dadirri. Considéré comme le cœur du programme et comme l'agent thérapeutique de ce dernier (Tasia, 2018b), le Dadirri prend la forme d'une méditation guidée au cours de laquelle les membres sont invités à écouter un texte d'Ungunmerr-Baumann (une Aborigène de la région de Daly River ${ }^{9}$ ) très graphique et dont le contenu transpire l'aboriginalité, et à se projeter mentalement dans l'image ainsi générée. Par ce processus, les membres sont donc plongés dans un exercice "d'imagination active " de type jungien (voir von Franz, 1976) dont l'objectif est d'être transporté au milieu du bush ${ }^{10}$, en situation d'initié du Dreaming. L'exercice dure une petite dizaine de minutes et est directement suivi par un échange sur les sensations ressenties par chacun. L'ensemble du protocole permet aux membres d'établir un lien cognitif et affectif puissant entre leur démarche de DP et celle d'une connexion retrouvée avec "leur " culture, ré-enchantant du même coup leur monde (Susswein \& Tasia, à paraître). À nouveau, il importe peu que la technique du Dadirri soit originellement pratiquée par les Aborigènes du Territoire du Nord (Ungunmerr-Baumann, 1993) ; seul compte le sens qu'offre la technique, par ses accents culturels prononcés, à la démarche de QS. C'est ce dont témoignent les propos suivants, tenus par une membre active du groupe :

Pour moi, [Dadirri] c'est un vrai cadeau, un cadeau très important. Celui-ci devrait être apprécié au quotidien. Car cela m'a aidé... [Grâce à lui,] je suis devenue une femme noire fière et forte ; je me suis trouvée moi-même. [Aujourd'hui] je sais qui je suis. Savoir que nous sommes culturellement connectés à ce territoire. . . c'est un cadeau ! Etre née aborigène est une vraie bénédiction ; ce n'est pas quelque chose dont nous devrions être honteux. Tu sais, honnêtement, cette connexion avec nos magnifiques territoires et notre culture, c'est le plus merveilleux des cadeaux.

Enfin, (c) certains membres n'hésitent pas à perpétuer ce qui est a été entamé au sein du Gamarada, en dehors de celui-ci. Ces derniers cherchent alors activement à obtenir des "signes ", à collecter des messages émis par l'environnement leur étant tout particulièrement destinés et leur indiquant alors " le chemin à suivre " (the path to follow) :

Tu sais, tout est là, dehors... Il suffit de suivre les signes. [Ça] peut être n'importe quoi : un oiseau qui chante ou une personne chuchotant quelque chose d'important, en passant sur le chemin des courses. N'importe quoi... [il] suffit juste d'être alerte (to be alert)... C'est comme ça que ça marche pour moi en tout cas. C'est ma façon à moi de trouver le chemin de ma culture (to find my way home to my culture).

\footnotetext{
${ }^{9}$ Pour plus d'informations, voir Ungunmerr-Baumann (1993).

10 Nom usuel donné au désert australien.
}

Pratiquement, ces signes sont constitués d'éléments banals du quotidien (comme, par exemple, le fait d'être témoin d'une branche d'arbre qui tombe, voir un oiseau qui se pose sur la chaise que l'on convoitait, rêver d'un être cher, etc. $)^{11}$; pragmatiquement, elles ont pour objectif d'orienter l'action future de l'individu en posant comme nécessité que cette dernière s'inscrive dans le registre du sacré par la réalisation d'une liaison entre ce même individu et son " destin ". Autrement dit, ces signes fonctionnent comme des indices émis par la " sémiose " constitutive de l'environnement (Kohn, 2017), permettant de relier - sur le plan mystique - l'individu à ce dernier. En ce sens, cette quête de signes s'inscrit dans la logique d'interconnectivité totémique propre à la cosmologie du Dreaming (Glaskin, 2015 ; Glowczewski, 1991a ; Poirier, 2005).

\section{Conclusion}

Par la conjoncture de trois éléments socio-anthropologiques (contexte, dispositions, techniques), les membres du Gamarada parcourent leur " voyage thérapeutique "; ils cheminent vers la transcendance de leur état malheureux et, du même coup, vers une autre version d'eux-mêmes, " renforcée " (empowered) par " leur " culture retrouvée. En cela, ils font preuve de résilience : ils adaptent leurs comportements à la situation difficile qui est la leur (Tasia, 2018a). Mais une telle démarche ne peut trouver son sens que dans la réappropriation, sous des modalités nouvelles, du Dreaming. Comme nous venons de le montrer, c'est par l'utilisation de diverses pratiques hétéroclites (narration de récits mythiques, pratique du Dadirri et quête de signes) que cela est rendu possible ; c'est n'est qu'une fois transformé, modulé tant dans son contenu propre que dans ses façons d'être (ré)actualisé de manière rituelle que le Dreaming devient, pour les membres du Gamarada, un substrat culturel dans lequel ces derniers peuvent espérer trouver de quoi nourrir leurs attentes. De manière concomitante, ce n'est qu'en absorbant toutes ces variations que le Dreaming parvient à se maintenir comme élément mythico-pratique de plein droit parmi ces populations aborigènes urbaines. Par conséquent, c'est à l'ensemble du système qu'il faudrait étendre le principe de résilience susmentionné. En effet, comme nous l'évoquions au début de ce texte et comme de nombreux auteurs l'ont démontré (Dussart, 2000 ; Eickelkamp, 2011 ; Glowczewski, 1991a ; Poirier, 2005), le Dreaming n'a jamais été une entité culturelle statique ; ce " passé mythologique [qui] est vivant et pertinent dans le présent " (Berndt \& Berndt, 1965, p. 188), est intrinsèquement dynamique et résilient. Tout autant constitué que constitutif des rituels, des histoires cosmogoniques, des parcours sacrés et des liens totémiques qui le font vivre, il entretient avec les populations aborigènes du pays un lien de production et de reproduction particulièrement actif. De par son ancrage pragmatique dans la géographie du pays (the dreaming tracks) et dans le corps social des sociétés aborigènes (the Lore), le Dreaming est pratiquement indissociable, pour ces sociétés, d'une " philosophie de vie " (Stanner, 1979, p. 36). Il n'y a, dès lors, plus rien d'étonnant à constater la plasticité historique du Dreaming tant le destin de cette cosmologie est intimement lié celui de ceux qui la font vivre : les Aborigènes. Or, avec une population contemporaine principalement urbaine et péri-urbaine ${ }^{12}$, et donc

\footnotetext{
${ }^{11}$ Dans un tout autre registre, le travail remarquable sur le " mode d'existence " des morts de Vinciane Despret (2015) a su très justement capter la nature épistémique " apostrophique " de ce genre de "signes " que les morts font parvenir aux vivants pour établir une communication avec ces derniers. Fragiles, changeants, métaphoriques et même parfois absurdes, sont ce qui les caractérisent le mieux ; et c'est par ces caractéristiques qu'ils sont reconnus comme signes par les acteurs y faisant référence.

${ }^{12}$ Selon les statistiques officielles, en juin 2016, la population aborigène d'Australie s'élevait à 798400 individus, et constituait à peu près $3,3 \%$ de la population totale du pays. Parmi ceux-ci, $81,4 \%$ vivent en ville, en région urbaine ou en région péri-urbaine. Seuls $18,6 \%$ des Aborigènes vivent donc à (longues) distances des centres urbains (ABS, 2016).
} 
déconnectée de ses territoires originels - là où le Dreaming traditionnel prend tout son sens -, les Aborigènes d'Australie se doivent de se réinventer sans cesse culturellement sous peine de voir leurs cultures disparaître, englouties par le capitalisme néolibéral occidental (Altman \& Hickson, 2010). Pour ce faire, ces derniers doivent faire preuve d'une grande créativité vis-à-vis, entre autres, de leur corpus mythico-pratique. Et c'est ce que font notamment les membres du Gamarada en entreprenant une QS basée sur cet ensemble de pratiques et de discours hétéroclites. De fait, comme nous le rappelle Torrance, " la quête spirituelle est le processus créatif par excellence, le processus par lequel les humains se réinventent continuellement [...] trouvant son expression dans les histoires incorporées qui composent les rituels et les mythes " (1994, p. 57). Ici encore, par conséquent, rien d'étonnant à voir la résilience de certains individus particulièrement volontaires prendre, en acte, la forme d'une QS s'inscrivant dans l'épistémologie des cultures dont ils sont issus puisqu'au fond, c'est par un effort de créativité tant au niveau individuel que culturel que ces derniers parviennent à améliorer leurs existences et combattre la souffrance $^{13}$; c'est par cet effort de créativité que le processus thérapeutique dans lequel ils sont engagés se voit opérationnalisé.

\section{Remerciements}

Je remercie les deux relecteurs anonymes pour leurs commentaires et critiques de grandes qualités. Je remercie également le Fond national de la recherche scientifique (FRS-FNRS) dont le financement a permis la réalisation du travail de thèse à la base de cet article.

\section{Références}

Australian Bureau of Statistics (ABS) (2016). Aboriginal and Torres Strait Islander Population, 2016 census data summary. Retrieved from https://www.abs.gov.au/ ausstats/abs@.nsf/Lookup/by\%20Subject/2071.0 2016 Main\%20Features Aboriginal\%20and\%20Torres\% 20Strait\%20islander\%20Population\%20Article 12

Australian Institute of Health and Welfare [AIHW] (2015). The Health and Welfare of Australia's Aboriginal and Torres Strait Islander Peoples. Canberra: Australian Institute of Health and Welfare.

Altman, J., \& Hickson, M. (Eds.). (2010). Culture Crisis: Anthropology and Politics in Aboriginal Australia. Sydney: University of New South Wales Press.

Atkinson, J., Nelson, J., \& Atkinson, C. (2010). Trauma, transgenerational transfer and effects on community wellbeing. Working Together, 135, 135-144.

Atkinson, J. (2002). Trauma Trails, Recreating Song Lines: The Transgenerational Effects of Trauma in Indigenous Australia. North Melbourne: Spinifex Press.

Attwood, B. (2005). Telling the Truth about Aboriginal History. Crows Nest. N.S.W Allen \& Unwin.

Berndt, R. M., \& Berndt, C. H. (1965). The World of the First Australians; an Introduction to the Traditional Life of the Australian Aborigines. Chicago: University of Chicago Press.

Berndt, R. M. (1947). Wuradjeri magic and 'Clever Men'. Oceania, 17(4), 327-365.

Burbank, V. K. (2011). An ethnography of stress: the social determinants of health in aboriginal australia. New York: Palgrave Macmillan.

Cowlishaw, G. (2010). Mythologising culture: part 1: desiring aboriginality in the suburbs. The Australian Journal of Anthropology, 21(2), 208-227.

Cowlishaw, G. (2011). Mythologising culture: part 2: disturbing aboriginality in the suburbs. The Australian Journal of Anthropology, 22(2), 170-188.

Cyrulnik, B. (2002). Un merveilleux malheur. Paris: Odile Jacob.

Dean, C. L. (1996). The Australian aboriginal dreamtime: an account of its history, cosmogenesis, cosmology and ontology. Geelong, Vic: C Dean.

Despret, V. (2015). Au Bonheur des morts : récits de ceux qui restent. Paris: Editions La Découverte.

Dussart, F. (1988). Warlpiri Women's Yawulyu Ceremonies: A Forum for Socialization and Innovation (Doctoral Dissertation). Canberra: Australian National University.

Dussart, F. (2000). The Politics of Ritual in an Aboriginal Settlement: Kinship, Gender, and the Currency of Knowledge. Washington, DC, London: Smithsonian Inst Press.

Ehrenberg, A. (2012). La Société Du Malaise. Paris: Odile Jacob.

Ehrenberg, A. (2018). La Mécanique Des Passions. Cerveau, Comportement, Société. Paris: Odile Jacob.

\footnotetext{
13 Dans un registre similaire, Tobie Nathan a su montrer en quoi la dimension créative des dispositifs psychothérapeutiques propres aux sociétés non-occidentales jouait un rôle primordial dans l'efficacité de celles-ci sur leur public respectif (Nathan, 2001 ; Nathan \& Stengers 2012).
}

Eickelkamp, U. (2011). Sand Storytelling: tis social meaning in Anangu children's lives. In U. Eickelkamp (Ed.), Growing up in Central Australia: new anthropological studies of aboriginal childhood and adolescence (pp. 103-130). New York: Berghahn Books.

Elkin, A. P. (1977). Aboriginal Men of High Degree. Brisbane: University of Queensland Press.

Elkin, A. P. (1986). The Australian Aborigines. North Ryde, Australia, London: Angus and Robertson.

Ferenczi, S. (2010). Le traumatisme. Paris: Payot \& Rivages.

Freud, S. (2010). Au-delà du principe de plaisir. Paris: Payot \& rivages.

Freud, S. (2015). Totem et tabou. Paris: Flammarion.

Garnoussi, N. (2013). Des glissements du spirituel au " psy " : Entre quête de sens et quête de santé dans le contexte de la sécularisation avancée. Archives de sciences sociales des religions, $163,63-82$.

Glaskin, K. (2015). Dreams, Perception, and Creative Realization. Topics in Cognitive Science, 7(4), 664-676.

Glowczewski, B. (1989). La Loi du rêve approche topologique de l'organisation sociale et des cosmologies des aborigènes australiens (Doctoral Disseration) Lille 3, Lille.

Glowczewski, B. (1991a). Du rêve a la loi chez les aborigènes : mythes, rites et organisation sociale en Australie. Paris: Presses Universitaires de France.

Glowczewski, B. (1991b). Entre Rêve et mythe : Roheim et les Australiens. L'Homme, 31(118), 125-132.

Glowczewski, B. (2004). Rêves en colère avec les Aborigènes australiens. Paris: Plon.

Grieves, V. (2009). Aboriginal Spirituality: Aboriginal Philosophy, the Basis of Aboriginal Social and Emotional Wellbeing. Casuarina, N.T Cooperative Research Centre for Aboriginal Health.

Henry, R. (2014). Performing Place, Practising Memories: Aboriginal Australians, Hippies and the State. New York: Berghahn Books.

Keen, I. (Ed.). (1988). Being Black: Aboriginal Cultures in "settled" Australia. Canberra: Aboriginal Studies Press.

Kohn, E. (2017). Comment pensent les forêts. Bruxelles: Zones sensibles.

Kowal, E. (2017). Descent, Classification and Indigeneity in Australia. In K. McGavin \& F. Fozdar (Eds.), Miwed Race Identities Australia, New Zealand and the Pacific Islands (pp. 19-35). New York: Routledge.

Lavarch, M. (1997). Bringing Them Home: Report of the National Inquiry into the Separation of Aboriginal and Torres Strait Islander Children from Their Families. Sydney: Human Rights and Equal Opportunity Commission.

Lambert-Pennington, K. (2012). 'Real Blackfellas': constructions and meanings of urban indigenous identity. Transforming Anthropology, 20(2), 131-145.

Macintyre, S., \& Clark, A. (2005). The History Wars. Melbourne: Vision Australia Information and Library Service.

Marquis, N. (2014). Du bien-être au marché du malaise : la société du développement personnel. Paris: Presses universitaires de France.

Marquis, N. (2016). Performance et authenticité, changement individuel et changement collectif : une perspective sociologique sur quelques paradoxes apparents du "développement personnel". Communication \& Management, 13(1), 47-62.

McComsey, T. (2013). Seeing and Being Seen: Aboriginal Community Making in Redfern (Doctoral Dissertation). Manchester: University of Manchester.

Moses, A. D. (2012). Genocide and settler society: frontier violence and stolen indigenous children in Australian history. New-York: Berhahn Books.

Murphy, F. (2011). Archives of Sorrow: An Exploration of Australia's Stolen Generations and Their Journey into the Past. History and Anthropology, 22(4), 481-495.

Nathan, T. (2001). L'influence qui guérit. Paris: Odile Jacob.

Nathan, T., \& Stengers, I. (2012). Médecins et sorciers. Paris: La Découverte.

Neuenfeldt, K. (1998). The quest for a'Magical Island': the convergence of the didjeridu, aboriginal culture, healing and cultural politics in new age discourse. Socia Analysis: The International Journal of Social and Cultural Practice, 42(2), 73-102.

O'Loughlin, M. (2009). A Psychoanalytic Exploration of Collective Trauma among Indigenous Australians and a Suggestion for Intervention. Australasian Psychiatry, (1), 33-36.

Pattel-Gray, A. (Ed.). (1996). Aboriginal Spirituality: Past, Present, Future. Blackburn, Vic: Harper Collins Religious.

Poirier, S. (2005). A World of Relationships: Itineraries, Dreams, and Events in the Australian Western Desert. Toronto, Buffalo: University of Toronto Press.

Povinelli, E. (2002). The Cunning of Recognition: Indigenous Alterities and the Making of Australian Multiculturalism. Durham: Duke University Press.

Povinelli, E. (2010). Indigenous politics in late liberalism. In J. Altman \& M. Hickson (Eds.), Culture Crisis: Anthropology and Politics in Aboriginal Australia (pp. 17-31). Sydney: University of New South Wales Press.

Reece, B. (1974). Aborigines and Colonists: Aborigines and Colonial Society in New South Wales in the 1830 s and 1840 s. Sydney: Sydney University Press.

Requilé, É. (2008). Entre souci de soi et réenchantement subjectif, sens et portée du développement personnel. Mouvements, 2, 65-77.

Reynolds, H. (1979). Aborigines and Settlers: The Australian Experience, 1788-1939. Stanmore: NSW: Cassell Australia.

Rimke, H. (2012). The Culture of Therapy: Psychocentrismin Everyday Life. In D. Brock, R. Raby, \& T. Mark (Eds.), Power and Everyday Practices (pp. 182-202). Toronto: Nelson Education.

Spencer, B., \& Gillen, F. (1898). The Native Tribes of Central Australia. London: Macmillan and Co limited.

Stanner, W. E. H. (1979). White Man Got No Dreaming. Canberra: Australian National University Press.

Susswein, R., \& Tasia, E. (2019). S'initier au merveilleux. Contribution à une socioanthropologie de l'enchantement à partir de l'étude comparée du " Gamarada » et de la " Communication animale intuitive ". EspacesTemps (à paraître).

Sutton, P. (2010). Aboriginal spirituality in a new age: aboriginal spirituality in a new Age. The Australian Journal of Anthropology, 21(1), 71-89. 
Stratton, J. (2011). Uncertain Lives: Culture, Race and Neoliberalism in Australia. Cambridge Scholars Publishing.

Tasia, E. (2016). Être étonné ? Lorsque le malentendu et l'angoisse nous ancrent dans le terrain. Civilisation, 65(1-2), 127-144.

Tasia, E. (2018a). Au-delà du rêve : ethnographie affective du processus de résilience des Aborigènes de Sydney (Australie). Tsantsa, 23, 14-23.

Tasia, E. (2018b). Du traumatisme à la résilience. Etude de cas socio-anthropologique d'un dispositif thérapeutique indigène de la banlieue centrale de Sydney (Australie) (Doctoral Dissertation). Bruxelles: Université Libre de Bruxelles.

Torrance, R. (1994). The Spiritual Quest: Transcendence in Myth, Religion, and Science. Berkeley: University of California Press.
Ungunmerr-Baumann, M.-R. (1993). Dadirri. In J. Hendriks \& G. Hefferan (Eds.), A Spirituality of Catholic Aborigines and the Struggle for Justice (pp. 34-37). Kangaroo Point, Brisbane Qld: Aborigines \& Torres Strait Islander Apostolate.

von Franz, M. L. (1976). Confrontation with the Collective Unconscious. Los Angeles: CG Jung Cassette Library.

Wolfe, P. (1991). On being woken up: the dreamtime in anthropology and in Australian settler culture. Comparative Studies in Society and History, 33(2), 197-224.

Zsolnai, L., \& Flanagan, B. (2019). The Routledge International Handbook of Spirituality in Society and the Professions. New-York: Routledge. 
